\title{
Background Paper
}

\section{Inductive foraging: Improving the diagnostic yield of primary care consultations}

\author{
Norbert Donner-Banzhoff $M D, M H S c^{1}$ \& Ralph Hertwig PhD \\ ${ }^{1}$ Department of General Practice, University of Marburg, Germany, and ${ }^{2}$ Department of Adaptive Rationality, Max-Planck-Institute for \\ Human Development, Berlin, Germany
}

KEY MESSAGE:

- Hypothetico-deductive reasoning has become the orthodox descriptive and normative model. However, this is not the whole story.

- 'Inductive Foraging' is a highly efficient data gathering strategy for generalist practice, when a large number of aetiologies have to be considered.

- Research from clinical reasoning research, cognitive psychology and education, support the concept of 'inductive foraging'.

\begin{abstract}
Background: Physicians attempting to make a diagnosis arrive at specific hypotheses early in their encounter with patients. Further data are collected in the light of these early hypotheses. While this hypothetico-deductive model has been accepted as both a description of physicians' data gathering and a norm, little attention has been paid to the preceding stage of the consultation. Hypothesis: It is suggested that 'inductive foraging' is a relevant and appropriate mode of data acquisition for the first part of the patient encounter.

Methods: Research evidence from cognitive psychology and medical reasoning research is discussed.

Results: With inductive foraging, 'pattern failure' rather than 'pattern recognition' is the mode of discovery. Largely, guidance should be left to the patient to lead the clinician into areas where departures from normality are to be found. This is in contrast to active and focused 'deductive inquiry,' which should be used only after most aetiologies, but a few have eliminated.

Implication: Especially when the prevalence of serious disease is low, and a wide range of diagnoses must be evaluated, such as in General Practice, inductive foraging is a rational and efficient diagnostic strategy. Previously, too little attention has been paid to the initial stage of the consultation. Premature closure at this point may result in diagnostic error.
\end{abstract}

Keywords: decision making, uncertainty, cognition, perception, judgement, diagnosis, general practice, family practice

\section{INTRODUCTION}

Medical practitioners' reasoning and data collection is often framed as hypothetico-deductive (1). According to this view, clinicians form a hypothesis based on their first impression early in the consultation with the patient. More hypotheses, i.e. possible diagnoses, are added, but their number is usually limited. Further data collection by history taking and physical examination is guided by these early hypotheses. Once the likelihood of a diagnosis has reached a sufficiently high threshold, data collection is stopped, and either treatment is started or further testing is performed.

Premature closure is said to occur when clinicians stop their data collection too early and, as a consequence, miss important information. This has been identified as an important source of diagnostic error (2-4). In this view, premature closure can happen only after the first hypothesis has arisen in the clinician's mind. To date, little attention has been paid to the period before a hypothesis has been formed. We would suggest that at

Conference Presentations: NDB presented parts of this article at the COGITA working group of the European General Practice Research Network, Zürich, Switzerland, October 2010

Correspondence: Norbert Donner-Banzhoff, Department of General Practice, University of Marburg, Karl-von-Frisch-Str. 4, D-35043 Marburg, Germany. E-mail norbert@staff.uni-marburg.de 
this, the hypothesis-generation phase is of crucial importance for the diagnostic outcome of the consultation especially in General Practice.

\section{INDUCTIVE FORAGING}

We are suggesting here that a stage to be called 'inductive foraging' precedes hypothesis formation. Ideally, the space of possible symptoms is searched with an open mind on the side of the physician. To what degree clinicians are able to restrain themselves at this stage, determines the number and appropriateness of diagnostic hypotheses considered for an individual patient. Thus, the foraging stage is an important part of the diagnostic process and deserves more attention from clinicians, researchers, and educators. We contend that sufficient evidence is available to make some tentative recommendations regarding tactics to be used during inductive foraging.

During the inductive foraging stage of the consultation, the patient leads the clinician to areas that are unusual or worrisome. Here, the predominant diagnostic strategy may be one of 'pattern failure' rather than 'pattern recognition' (5). Lewis likens the situation to an LCD monitor screen the working of which one is hardly aware of. However, if a pixel is not working, one's eye is inevitably drawn to it with the resulting feeling that something is wrong. Accordingly, in the consultation there is often a stage when single pieces of information cannot yet be matched to a pattern. This will stimulate further probing of unusual phenomena until the range of possibilities is narrowed into a specific hypothesis. Another possibility is the impression, that something does not 'feel right,' or that a particular patient 'is not herself' (see below for meningococcal infection as an example). As long as no departure from 'normal' is noticed, the default is the assumption that there is no pathology deserving further investigation, at least as long the problem space has been foraged to a sufficient extent. Inductive foraging has universal application even beyond medical diagnosis (6), but it is especially suitable for primary care where the prevalence of serious disease is low but a wide range of possibilities must be explored.

\section{EVIDENCE FROM CLINICAL REASONING RESEARCH}

Although, our suggestion of 'inductive foraging' is novel as a diagnostic strategy, there is indirect evidence in the literature supporting the concept. From his analysis of Dutch general practitioners (GPs) working with simulated patients, Ridderikhof (7) concluded that inductive reasoning is the predominant mode of data collection and reasoning. In his view, information from the patients 'serves the process of evoking hypotheses rather than testing them.' Ridderikhof highlighted the speculative and erratic nature of the hypothesis-generating process, which he considered at odds with professional accountability and the scientific foundation of medical practice.

Bordage et al. (8) saw what they call a 'responsive mode of enquiry' in a more positive light. They encouraged clinicians to alternate their thinking between a deterministic mode, in which questions related to a specific hypothesis are being asked, and a responsive mode, paying attention to information offered by the patient.

The main conclusion drawn from the Medical Inquiry Project (1) is that a purely inductive method of data gathering is never used by physicians, but that hypotheses are formed early and guide further work-up. However, even in this study with standardized patients diagnostic accuracy was associated with the amount of cues acquired. In some cases, withholding hypothesis generation until sufficient data had been obtained apparently resulted in better diagnosis. A strategy of inductive reasoning was associated with diagnostic success in a think-aloud study of paper cases for medical students and experienced gastroenterologists (9).

Granier et al. report the retrospective accounts of Welsh GPs managing children with meningococcal disease (10). This is an interesting case study since the condition is so rare that GPs cannot gather sufficient experience diagnosing it. Instead, they were using departures from an assumed normal state as an indication of a serious problem. The behaviour of parents provided cues in some cases, such as a mother of three children, usually coping well, suddenly bursting into tears. Another point of reference was the image of benign infection. Children being lethargic, not interacting with either their parents or the GPs, or crying in an unusual way presented exceptions to an assumed normality of benign, self-limiting infection. The matching of information to a positive pattern was not yet possible at that stage, so physicians' reasoning rather took the form of pattern failure. This still resulted in optimal decisions, i.e. immediate transfer to hospital. A study of Danish family physicians diagnosing neglect and abuse in children showed similar results (11). These findings have led to the postulate that a family of discrepancy heuristics underlies a large part of diagnostic assessment in primary care practice (12). Their frame of reference may be previous experience with a particular patient (within-person comparison), or typical behaviour of similar patients (between-person comparison). In some European countries, GPs are encouraged to act on their gut feelings or their sense of alarm (13-14).

\section{INSIGHTS FROM COGNITIVE PSYCHOLOGY}

Using the background of Popper's theory of falsification (15) as one benchmark within the context of discovery, numerous investigators have deplored the seemingly irrational behaviour of subjects confronted with rule 
discovery or diagnostic tasks. Instead of aiming to refute their hypotheses by searching for disconfirming evidence, most people seek information that confirms the idea (hypothesis) they have formed. Accordingly, clinicians seek abnormal symptoms or findings that fit their hypotheses. For rational inference, however, one should evaluate a single piece of information, such as fever, across hypotheses, and thus draw conclusions from the absence as well as from the presence of a signal.

Klayman and $\mathrm{Ha}$, however, have suggested that a confirmative or positive test strategy can be appropriate, especially 'when concrete, task-specific information is lacking, or cognitive demands are high' (16). According to this strategy, individuals search for and examine instances in which the target behaviour, property, or event is expected to occur, as opposed to searching for instances where it is not expected to occur under the hypothesis entertained. They reason that under certain circumstances positive evidence is more likely to prove a hypothesis wrong. This is the case in probabilistic settings and where the target event is rare. The General Practice consultation is a good example. Here, the prevalence of serious disease is low and related pathological findings are rare. For each patient, the most likely hypothesis is thus absence of serious disease. Accordingly, it is the presence of an abnormality that disconfirms the most likely hypothesis. The presence of an abnormality is more informative than confirmation of its absence. However, the reverse is true if the probability of the target category, i.e. disease, is high. In that case, the hypothesis is efficiently tested also by negative evidence, i.e. absence of findings $(16,17)$.

Human beings find it difficult to evaluate isolated information, especially if presented in a quantitative way. Often only the provision of an anchor for comparison enables individuals to evaluate a statement. There is extensive research showing how anchoring, i.e. comparing a stimulus with a frame of reference, can mislead a decision-maker if taken to a different context (18-20). However, despite this line of research, one should not forget that comparing a particular finding with a frame of reference provides a powerful and ubiquitous heuristic in human and clinical decision-making. What constitutes information in the clinical encounter can only be defined by comparison with a reference. Pain experienced by a patient with chronic rheumatoid arthritis on one of her good days can be devastating for a person who has not had musculoskeletal problems before. At this stage, therefore, it makes sense to leave the initiative to the patient to lead the physician to problem areas, i.e. areas where well-being or function differs from normal, which can thus provide clinically relevant information. In this way, the clinician can make productive use of the frame of reference defined by the patient.

Interestingly, the developers of a computer-based general medical expert system concluded that one of the limitations of their product is the inability to take a broad perspective of a case presented. The system would suffer from a 'tunnel vision,' and only after having arrived at specific diagnosis would the links to their sophisticated data base provide satisfactory diagnostic results $(21,22)$. This example from applied artificial intelligence underlines the importance of the foraging stage, but also the difficulty delegating the task to a machine.

\section{DEDUCTIVE INQUIRY, INDUCTIVE FORAGING AND PREMATURE CLOSURE}

By 'premature closure,' authors usually mean the end of a phase of specific hypotheses evaluation. We suggest the term 'deductive inquiry' for this phase since reasoning goes from propositions (hypothesis) to data gathered by history taking or examination of the patient. We contend that premature closure can also occur earlier than that, marking the end of the preceding inductive foraging phase and starting 'deductive inquiry' prematurely (see Figure 1). This may result in missing relevant diagnostic information. Instead, patients should be encouraged to elaborate on symptoms or, more generally, on deviations from their 'normal' state and associated concerns. This strategy promises to yield a rich set of information and does not foreclose the retrieval of information not expected by the clinician. It amounts to a positive test strategy in so far as the patient can be expected to focus on noteworthy occurrences of unusual events, such as symptoms and concerns. In contrast, adopting a question-and-answer-mode early on risks that the patient volunteers only the information that has been explicitly asked for.

There seem to be compelling reasons for physicians to interrupt their patients early in the consultation $(23,24)$. To expedite the consultation and thereby save time is an obvious motive for busy clinicians. A more subtle consideration refers to re-interpretation of information in view of a diagnostic hypothesis. Once

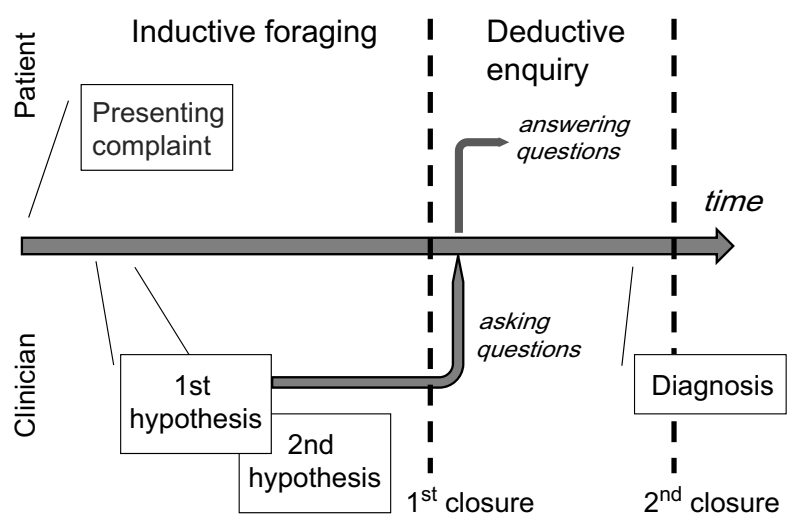

Figure 1. Stages of the clinical encounter and related modes of inquiry. 
physicians have arrived at their first hypothesis, further information provided by the patient is seen in a '(dis-) confirmatory' light, i.e. whether or not the information is relevant to the hypothesis entertained by the practitioner. A large amount of information can be discarded as not contributing. A messy picture suddenly looks ordered. However, at this early stage there is a risk of too greatly reducing the range of possible explanations. To conclude that relevant diagnoses have been excluded with acceptable probability, problem areas must be explored sufficiently. The order in which this should happen is best left to the patient. Given enough time, they will lead the clinician like 'a sniffer dog on a leash' (Anonymous GP, Basle [Switzerland]. Personal Communication. 2009) to areas where information can be found.

\section{CASE STUDY}

The efficiency of inductive foraging as a strategy of data acquisition is illustrated by the case of a 52-year-old female patient presenting with tiredness of three months' duration (Figure 2). A vast number of diseases can cause the symptom. Among these, defined somatic aetiologies are rare. Psychological disorders are more frequent, and often no specific cause can be found. Given enough time, this patient volunteers a history of pain in the frontal part of her neck and flu-like symptoms six months ago for which she had not sought medical help. These unusual symptoms alert the physician to the possibility of thyroiditis and consecutive hypothyroidism, which was corroborated later. The graph illustrates the vast array of pieces of potentially useful information. Only small areas can be covered with direct active questioning or searching, as shown in the graph for vital signs, symptoms of depression and medication that can cause tiredness. In the example presented here, these are all negative. However, foraging under guidance of the patient leads the clinician to a very rare complaint that triggers specific investigations. Premature switching to a question-and-answer-mode would have resulted in a longer and less focused process, which would have delayed definite diagnosis considerably.

\section{AREAS FOR FUTURE RESEARCH}

Few studies have investigated diagnostic reasoning and decision making in real practice. Little attention has been given to the earlier stage of patient-physician

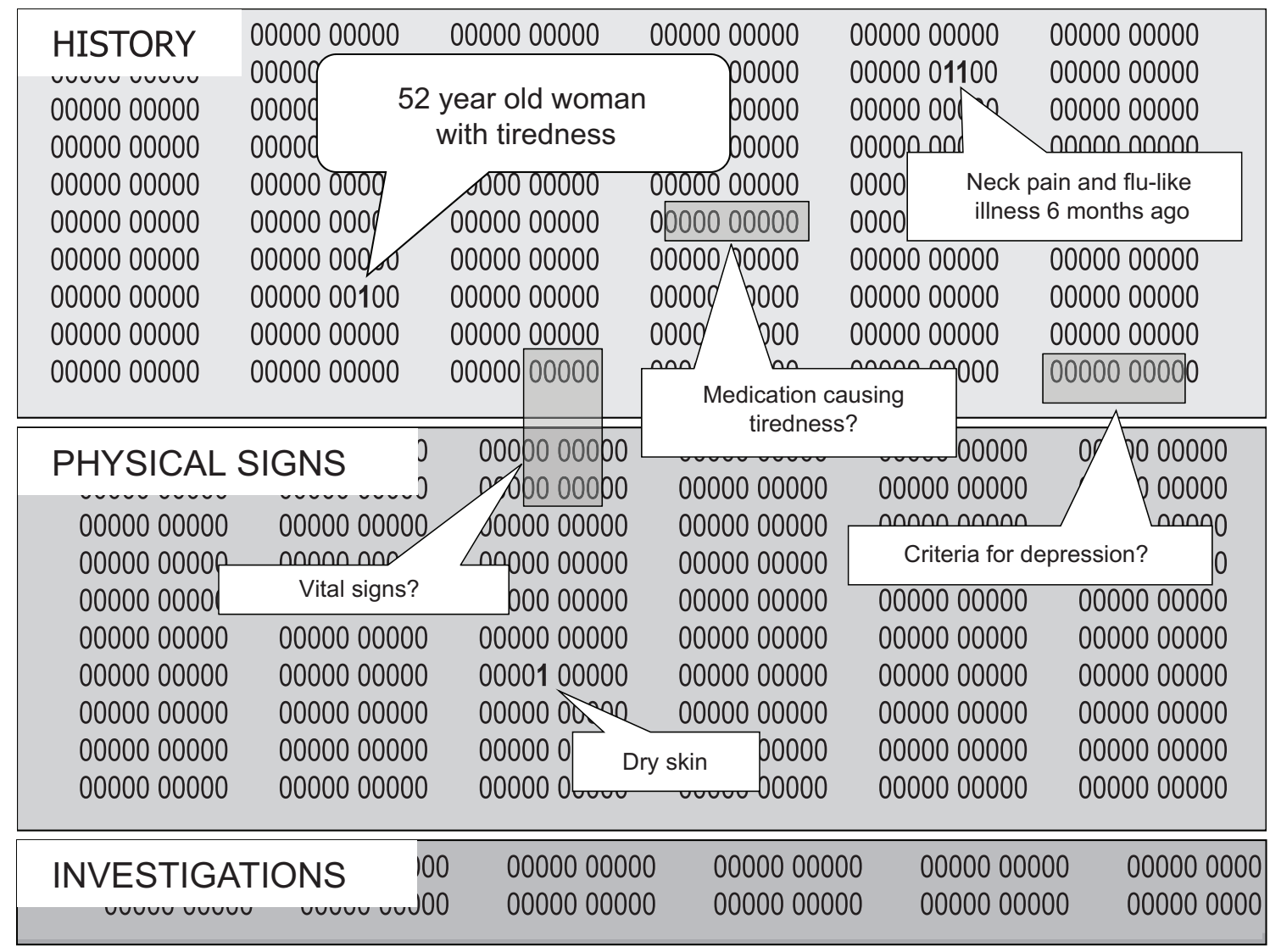

Figure 2. Case of a 52 year old woman presenting with tiredness. Graph shows space of potentially relevant information from history, physical signs and further investigations. ' 0 ', normal; ' 1 ', abnormal finding; grey rectangles, information actively asked for routinely. 
encounters. By definition, this stage is unstructured, and one cannot easily formulate specific rational strategies of inquiry. Moreover, the opening stage of an encounter depends on the context that cannot be captured by 'in vitro' studies based on paper cases or standardized patients. Therefore, future research should explore the dynamics of the 'inductive foraging' stage of the consultation, exploratory tactics used by clinicians, the responses they elicit from their patients, and whether these are associated with measures of diagnostic success.

\section{CLINICAL AND EDUCATIONAL RECOMMENDATIONS}

Health professionals need to be aware of the existence and value of the 'inductive foraging' stage described above. They should realize that giving patients sufficient time to elaborate on their complaints not only eases the patients' sense of distress (25), but is also likely to improve the diagnostic outcome of the consultation. Closed questions referring to the presence or absence of specific symptoms should be reserved for the 'deductive enquiry' stage. Instead, open questions encouraging further exploration and elaboration should be asked, if needed: How did it start? Have you noticed anything else? What else is different from usual? What about your everyday activities? With these kinds of questions clinicians will also be able to move the discourse back to 'inductive foraging' if premature closure has occurred by the patient. At the same time, clinicians should pay attention to their sense that perhaps something is not normal.

The considerations referring to inductive foraging are particularly relevant for the care of unselected populations, i.e. primary care or emergency department hospital settings (26). Here, complaints are often non-specific, and a large number of possible diagnoses must be screened; inductive foraging may be the most efficient way to achieve this. Prematurely advancing to hypothesis-guided deductive inquiry would inappropriately restrict the scope of possible explanations.

\section{CONCLUSION}

Hopefully, the concept of inductive foraging will not only have a descriptive value, but will also encourage physicians, teachers and students to reflect on how their diagnostic ideas arise. They will find that there is not necessarily a trade-off between patient-centred communication and effective diagnosis, because skilful inductive foraging can improve both.

Declaration of Interest: The authors report no conflicts of interest. The authors alone are responsible for the content and writing of the paper.

\section{REFERENCES}

1. Elstein AS, Shulman LS, Sprafka SA. Medical problem-solving: An analysis of clinical reasoning. Cambridge, MA: Harvard University Press; 1978.

2. Redelmeier DA. The cognitive psychology of missed diagnoses. Ann Intern Med. 2005;142:115-20.

3. Graber ML, Franklin N, Gordon R. Diagnostic error in internal medicine. Arch Intern Med. 2005;165:1493-9.

4. Voytovich AE, Rippey RM, Suffredini A. Premature conclusions in diagnostic reasoning. J Med Educ. 1985;60:302-7.

5. Lewis DM. Look before you leap. Br Med J. 2009; 338 http://www. bmj.com/rapid-response/2011/11/02/look-you-leap (accessed 16 June 2013).

6. Itti L, Baldi P. Bayesian surprise attracts human attention. Vision Res. 2009;49:1295-306.

7. Ridderikhoff J. Problem-solving in general-practice. Theor Med. 1993;14:343-63.

8. Bordage G, Grant J, Marsden P. Quantitative assessment of diagnostic ability. Med Educ. 1990;24:413-25.

9. Coderre S, Mandin H, Harasym PH, Fick GH. Diagnostic reasoning strategies and diagnostic success. Med Educ. 2003;37:695-703.

10. Granier S, Owen P, Pill R, Jacobson L. Recognising meningococcal disease in primary care: qualitative study of how general practitioners process clinical and contextual information. BMJ. 1998;316:276-9.

11. Lykke K, Christensen P, Reventlow S. This is not normal...-Signs that make the GP question the child's well-being. Fam Pract. 2008;146-53.

12. Donner-Banzhoff N. Wie stellt der Allgemeinarzt eine Diagnose? Z allg Med. 1999;75:744-9.

13. Stolper E, van Royen P, Dinant GJ. The 'sense of alarm' ('gut feeling') in clinical practice. A survey among European general practitioners on recognition and expression. Eur J Gen Pract. 2010;16:72-4.

14. Stolper E, Van de Wiel M, Van Royen P, Van Bokhoven M, Van der Weijden T, Dinant GJ. Gut feelings as a third track in general practitioners' diagnostic reasoning. J Gen Intern Med. 2011;26:197-203.

15. Popper KR. The logic of scientific discovery. London: Routledge; 2002.

16. Klayman J, Ha YW. Confirmation, disconfirmation, and information in hypothesis-testing. Psychol Rev. 1987;94:211-28.

17. Oaksford M, Chater N. Optimal data selection: Revision, review, and re-evaluation. Psychon Bull Rev. 2003;10:289-318.

18. Slovic P, Finucane ML, Peters E, MacGregor DG. The affect heuristic. Eur J Oper Res. 2007;177:1333-52.

19. Kahneman D, Miller DT. Norm theory-comparing reality to its alternatives. Psychol Rev. 1986;93:136-53.

20. Hsee CK, Loewenstein GF, Blount S, Bazerman MH. Preference reversals between joint and separate evaluations of options: $A$ review and theoretical analysis. Psychol. Bull. 1999;125:576-90.

21. Miller RA, Pople HE, Myers JD. Internist-I, an experimental computer-based diagnostic consultant for general internal medicine. New Engl J Med. 1982;307:468-76.

22. Spiegelhalter DJ, Knilljones RP. Statistical and knowledge-based approaches to clinical decision-support systems, with an application in gastroenterology. J Roy Stat Soc a Sta. 1984;147:35-77.

23. Marvel MK, Epstein RM, Flowers K, Beckman HB. Soliciting the patient's agenda-Have we improved? J Am Med Assoc. 1999; 281:283-7.

24. Rhoades DR, McFarland KF, Finch WH, Johnson AO. Speaking and interruptions during primary care office visits. Fam Med. 2001;33:528-32.

25. Howie JG, Porter AM, Heaney DJ, Hopton JL. Long to short consultation ratio: A proxy measure of quality of care for general practice. Br J Gen Pract. 1991;41:48-54.

26. Feufel MA. Bounded rationality in the emergency department. 2009; Wright State University, Dayton, Ohio (USA). Unpublished dissertation. 